\title{
A continuous time economic growth model with time delays in environmental degradation
}

\author{
Massimiliano Ferrara*• Luca Gori ${ }^{\dagger} \bullet$ Luca Guerrini ${ }^{\ddagger} \bullet$ Mauro Sodini $^{\S}$
}

August 22, 2017

\begin{abstract}
The aim of this work is to analyse the interplay between economic growth and environmental quality. Specifically, the article considers an economic growth model with optimising agents à la Ramsey where production negatively affects the environment. The novelty of this work is to assume that such a negative impact does occur with a certain time lag rather than instantaneously, as is commonly assumed in economic models. The existence of (discrete) time delays in this process may be a source of instability of the unique stationary equilibrium of the system and may cause the emergence of Hopf bifurcations.
\end{abstract} delays

Keywords Continuous time; Environmental degradation; Hopf bifurcation; Time

JEL Codes C62; O13; O41; Q22

AMS Codes $\quad 34 \mathrm{~A} 34 ; 65 \mathrm{P} 20 ; 70 \mathrm{~K} 50$

\section{Introduction}

Since the last decades there exists a burgeoning interest by theoretical and empirical economists in studying the interplay between economic growth and the environment (see Xepapadeas, 2005, for a detailed discussion on this issue). This is because also governments and policy makers are advocated of being alerted (sometimes driven by growing movements of public opinion) about the possible serious environmental degradation along the process of economic activity all around the world. Therefore, analysing issues related to sustainable growth from an academic point of view has become an important task. One of the first works that accounted for problems of environmental degradation in growth models is the seminal article of Day (1982), who considers a Solow-type growth model where pollution negatively affects production and fluctuations may arise because of the interplay between economic activity and environmental quality. Along this line, some works have focused on the role played by the environment in the production process, as

*M. Ferrara, Department of Law and Economics, Mediterranean University of Reggio Calabria, Via dei Bianchi, 2, I-89127 Reggio Calabria (RC), Calabria, Italy; e-mail addresses: massimiliano.ferrara@unirc.it.

${ }^{\dagger}$ L. Gori (corresponding author), Department of Political Science, University of Genoa, Piazzale E. Brignole, 3a, I-16125 Genoa (GE), Italy, e-mail: luca.gori@unige.it or dr.luca.gori@gmail.com, tel.: +39010209 95 03, fax: +390102095536.

${ }^{\ddagger}$ L. Guerrini, Department of Management, Polytechnic University of Marche, Piazza Martelli 8, I-60121, Ancona (AN), Italy, e-mail: luca.guerrini@univpm.it, tel.: +390712207055.

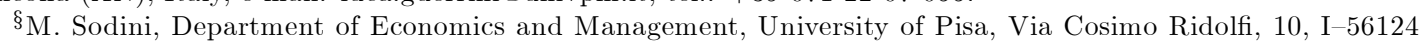
Pisa (PI), Italy, e-mail: mauro.sodini@unipi.it, tel.: +390502216 234, fax: +390502210603. 
pointed out in the so-called Green Solow model by Brock and Scott Taylor (2010), who relate the theoretical one-sector Solow growth model to the Environmental Kuznets Curve. Specifically, they build on a model that incorporates an exogenous abatement mechanism (technological progress in abatement) in the technology of production adopted by Solow (1956) and find that GDP growth causes an inverted U-shaped evolution in environmental quality (Environmental Kuznets Curve). Other works were developed to account for the behaviours of optimising agents in models that include environmental variables (see, for instance, Bovenberg and Smulders, 1995; Ayong Le Kama, 2001, Wirl, 2004; Antoci et al., 2011). Some of these contributions focus on the steady-state relationships between the main variables involved in the models, whereas others concentrate on the study of nonlinear dynamics when the environment affects production. For example, Antoci et al. (2011) find the possibility of the existence of persistent fluctuations in a continuous time model with optimising agents under the assumption of a technology of production that makes use of a free-access natural resource as an input. An alternative approach is that of assuming that an environmental variable (e.g., an index of environmental quality or pollution) directly enters the utility function of agents (John and Pecchenino, 1994; Zhang, 1999; Antoci, 2009). Even within this modelling approach it was shown the possibility of nonlinear dynamic outcomes. For example, Zhang (1999), by using a particular specification of the model of John and Pecchenino (1994), shows that the dynamics are described by a unimodal map.

We note that in discrete-time models the emergence of nonlinear dynamics is often related to the existence of a time lag between the economic activity (production or consumption) and its impact on environmental quality (Zhang, 1999; Antoci et al., 2016). Instead, in continuous time models, where the economic activity and its environmental damages are assumed to occur at the same time, nonlinear dynamics are generated by 1) particular nonlinear functional specifications of the evolution of environmental dynamics (D'Alessandro, 2007; Antoci et al., 2011) or 2) quite complex decision-making processes (optimisation problems with more than one control variable) of economic agents (Itaya, 2008; Antoci et al., 2011). The purpose of this article is to analyse the dynamics of a model in which production and allocation choices take place continuously, but there is a time delay between the economic activity and its impact on the environment (see, for example, Nadadur and Hollingsworth, 2015, for a survey on long-term effects of air pollution, and Mapanda et al. 2005, for a contribution on the long-term effects of irrigation using wastewater). ${ }^{1}$

From a modelling perspective, contributions on economic dynamics with time delays have existed since the thirties of the previous century (Kalecki, 1935). A part of this literature (Asea and Zak, 1999, Bambi, 2008) focused on macroeconomic business cycles by considering the role of gestation lags on investments (i.e., capital takes time to become productive). In addition, there is a branch of the literature that concentrated on the study of problems with the so-called vintage capital, that is models characterised by the coexistence of modern technologies and vintage technologies (see Boucekkine et al., 2011, and the literature cited therein). More recently, another part of the literature has been focusing on the study of dynamic microeconomic settings dealing with cobweb models (Gori et al., 2015) or monopoly or oligopoly problems (Matsumoto and Szydarowski, 2012).

By following the spirit of the models proposed by Wirl (1997) and Antoci et al. (2011, 2016), we assume that agents are unable to internalise the negative effects of their actions on environmental dynamics (negative externality) but they are able to perfectly foresee the time evolution and the values of economic variables. In order to focus on the role of the time delay on the dynamics of the model we consider a linear specification for the environmental evolution (similar to the one adopted by John and Pecchenino, 1994, but simpler than that used by Antoci et al., 2011) and only one control variable is introduced in the optimisation problem. As agents

\footnotetext{
${ }^{1}$ Amongst other things, these works include details on medical and or chemical reasons for which health injuries are delayed over time.
} 
consider the environment as an externality, there are no variables evaluated at future dates in the optimality conditions (as opposed to Bambi and Gori, 2014, where forward variables enter the first order conditions) and equilibrium dynamics are described by a system of three non-advanced (see Bambi, 2008, for terminology) differential equations with fixed delays.

On the one hand, in the absence of time lags of the impact of the economic activity on environmental dynamics, the system exhibits a unique fixed point which results to be saddle path stable (that is, the dynamics evolve on the two-dimensional stable manifold of the saddle, converging towards the stationary equilibrium) if the inter-temporal elasticity of substitution in consumption is sufficiently high (that is, the consumption of the private good and environmental degradation are complements) or if the inter-temporal discount rate is sufficiently low. However, when these conditions are not fulfilled, it is possible to find configurations of parameters such that the steady state of the system without delays undergoes a Hopf bifurcation. Such a bifurcation generates the birth of a limit cycle so that the dynamics of the system are characterised by cyclical behaviours.

On the other hand, it is interesting to note that even starting from a context of saddle path stability (in the absence of time delays), the existence of a time lag between the economic activity and the environmental damage can generate a Hopf bifurcation.

The rest of the article proceeds as follows. Section 2 sets up the model. Section 3 focuses on the study of the dynamics of the system. Section 4 outlines the conclusions.

\section{Set-up of the model}

The model we are herewith considering consists of an economy comprised of a continuum of identical agents whose population size is constant and normalised to unity. At every time $t \in$ $[0, \infty)$, the representative agent produces output $Y(t)$ by using the following Cobb-Douglas technology:

$$
Y(t)=K(t)^{\alpha}, \quad 0<\alpha<1
$$

where $K(t)$ is the stock of physical capital (the labour input is simply $L(t)=1$ ) employed in production.

Let us assume that the environmental quality at time $t$ is measured by index $E(t)$. The dynamics of $E(t)$ at time $t$ is determined by the level of such an index at the same time as well as by the economic activity that took place at an earlier date. Specifically, we assume that environmental degradation today is affected by the production activity, $Y(t-\tau)$, realised at time $t-\tau$, where $\tau \geq 0$ represents the time lag with which production negatively affects the environment. This assumption is made up to capture a peculiar aspect of the environmental degradation that is not closely related to current production but rather to what was produced in the (recent and distant) past (for instance, fine dust, generated from industrial production processes, cause a negative impact on health of humans over time; the same applies with the contamination of ground water due to pollution; another example concerns industrial discharges into the sea that contribute to create an unhealthy environment for the feeding of fish eventually causing damages for welfare of the human being). In this formulation, we assume that there is no dependency between the length of the time delay and the amount produced (as instead is the case in Boucekkine et al., 1998, who assume a technology characterised by a replacement rule of operating machines that generates state-dependent leads and lags). From a mathematical point of view, we use the following linear specification of environmental dynamics:

$$
\dot{E}(t)=\beta(\bar{E}-E(t))-\gamma \bar{Y}(t-\tau),
$$


where $E(t)$ is the time derivative of $E(t), \bar{Y}(t-\tau)$ is the economy-wide average output at time $t-\tau$ (which is taken as given by the representative agent), $\bar{E}>0$ represents the carrying capacity of the natural resource, $\beta>0$ measures the speed of convergence of the environmental quality towards the carrying capacity in the absence of production and $\gamma>0$ weights the negative impact of the production activity on environmental quality. It is important to note in the existing literature on environmental dynamics in growth models, several and often more complex specifications than the one adopted in (2) were used (D'Alessandro, 2007; Antoci et al., 2011). However, we chose a linear representation to focus on the role of the delay on the dynamics of the model.

The instantaneous utility at time $t, U(C(t), E(t))$, of the representative agent is a function of the consumption of the private good $C(t)$ and the environmental quality index $E(t)$. We specify this function by considering the following Constant Inter-temporal Elasticity of Substitution (CIES) formulation:

$$
U(C(t), E(t))=\frac{[C(t) E(t)]^{1-\sigma}}{1-\sigma},
$$

where $\sigma>0(\sigma \neq 1)$ denotes the inverse of the inter-temporal elasticity of substitution in consumption. From (3), it is clear that consumption $C(t)$ and environmental quality $E(t)$ enter multiplicatively in the utility function of the representative individual. This specification, in fact, allows us to capture both cases of complementarity $(\sigma<1)$ and substitutability $(\sigma>1)$ between consumption of the private good and environmental degradation. When consumption of the private good and the services provided by the environmental resource are complements (resp. substitutes), an individual wishes to reduce (resp. increase) private consumption to defend himself against environmental degradation. In the former case, in fact, the utility increases when consumption of the private good reduces as this generates an increase in the index of environmental quality. In the latter case, instead, the utility increases when consumption of the private good increases as this allows an individual to protect himself against the reduction in utility following environmental degradation. The use of air conditioners is a paradigmatic example of substitutability between private consumption and environmental quality. In fact, the marginal utility of cooling the interior of homes and offices decreases as long as global warming reduces. The expenses related to outdoor activities (if the sea is not polluted, one can rent a beach umbrella) or the use of bicycles as a means of transport (to go to work) are examples of complementarity between private consumption and environmental quality.

By assuming no depreciation of capital, the dynamics of $K(t)$ is given by the following differential equation

$$
\dot{K}(t)=Y(t)-C(t),
$$

where $K(t)$ is the time derivative of $K(t)$ and $Y(t)=K(t)^{\alpha}$ from (1).

The representative agent faces the following inter-temporal maximisation problem (for the sake of notational simplicity hereafter we omit the time subscript):

$$
\max _{C} \int_{0}^{+\infty} e^{-r t} \frac{(C E)^{1-\sigma}}{1-\sigma},
$$

subject to

$$
\begin{aligned}
\dot{K} & =K^{\alpha}-C, \\
\dot{E} & =\beta(\bar{E}-E)-\gamma \bar{Y}_{d}
\end{aligned}
$$


where the initial conditions $K(t), t \in(-\tau, 0]$ and $E(t), t \in(-\tau, 0]$ are given (we note that given the specifics of the optimisation problem, it is sufficient to consider a pointwise initial condition of $E$ at $t=0), K(t), E(t), C(t) \geq 0$ for every $t \in[0,+\infty), r>0$ is the inter-temporal discount rate and $\bar{Y}_{d}:=\bar{Y}(t-\tau)$. It is important to note that the representative agent takes the economy-wide average output $\bar{Y}_{d}$ and then the dynamics of $E$ as exogenously given in the maximisation problem. As there exists a continuum of agents of unitary mass, each agent does consider the impact of his choices on environmental dynamics as negligible. In addition, since agents are identical it holds ex post that $\bar{Y}_{d}=Y_{d}$.

\section{Optimality conditions and dynamics}

A special feature of this formulation of the problem is that as the dynamics of $E$ is taken as given by the representative agent, the only dynamic equation containing a time delay $(\dot{E})$ does not enter the optimisation problem (see Wirl, 1997). As we will later in this article, the unique delayed differential equation of the problem does not enter the optimality conditions and comes into play only when the equilibrium conditions of the economy $\left(\bar{Y}_{d}=Y_{d}\right)$ are imposed. We note that this kind of model differs from both models with vintage capital (Boucekkine et al., 1997, 2005) and models in which the system describing equilibrium dynamics can be returned to a system of differential equations without delays (Caulkins et al., 2010). The optimisation problem can then be tackled out through the use of the standard techniques of the maximum principle. The Hamiltonian function associated with the constrained maximisation of the inter-temporal utility function (5) of the representative agent is the following:

$$
H=\frac{(C E)^{1-\sigma}}{1-\sigma}+\lambda\left(K^{\alpha}-C\right) .
$$

where $\lambda$ is a co-state variable associated with the state variable $K$. The necessary and sufficient optimality conditions are then given by:

$$
\begin{aligned}
H_{C}^{\prime} & =0 \Longleftrightarrow \frac{E}{(C E)^{\sigma}}=\lambda, \\
\dot{K} & =K^{\alpha}-C, \\
\dot{\lambda} & =\lambda\left(r-\alpha K^{\alpha-1}\right),
\end{aligned}
$$

and the dynamics of $E$ is governed by (2). In addition, the following transversality condition should hold:

$$
\lim _{t \rightarrow \infty} \lambda K e^{-r t}=0 .
$$

This transversality condition is always fulfilled if $K$ and $\lambda$ converge towards a fixed point or a cycle.

By using logarithmic differentiation in (7), we find

$$
\frac{\dot{\lambda}}{\lambda}=(1-\sigma) \frac{\dot{E}}{E}-\sigma \frac{\dot{C}}{C}
$$

By imposing the equilibrium condition $\left(\bar{Y}_{d}^{\alpha}=Y_{d}^{\alpha}\right)$, the dynamic system becomes the following:

$$
\left\{\begin{array}{rl}
\dot{K} & =K^{\alpha}-C \\
\dot{E} & =\beta(\bar{E}-E)-\gamma K_{d}^{\alpha} \\
\dot{C} & =\frac{C}{\sigma}\left\{(1-\sigma)\left[\frac{\beta(\bar{E}-E)-\gamma K_{d}^{\alpha}}{E}\right]-\left(r-\alpha K^{\alpha-1}\right)\right\}
\end{array} .\right.
$$


Equilibria of system (8) are obtained by setting $\dot{K}=0, \dot{E}=0$ and $\dot{C}=0$. Then, we get the unique non-trivial equilibrium $\left(C_{*}, K_{*}, E_{*}\right)$, where

$$
K_{*}=\left(\frac{\alpha}{r}\right)^{\frac{1}{1-\alpha}}, \quad E_{*}=\bar{E}-\frac{\gamma}{\beta}\left(\frac{\alpha}{r}\right)^{\frac{\alpha}{1-\alpha}}, \quad C_{*}=\left(\frac{\alpha}{r}\right)^{\frac{\alpha}{1-\alpha}} .
$$

with

$$
\bar{E}>\frac{\gamma}{\beta}\left(\frac{\alpha}{r}\right)^{\frac{\alpha}{1-\alpha}} .
$$

The Jacobian matrix $J$ evaluated at $\left(C_{*}, K_{*}, E_{*}\right)$ leads to the following characteristic equation

$$
\operatorname{det}\left(A+B e^{-\xi \tau}-\xi I\right)=0,
$$

where $I$ is the identity matrix,

$$
A=\left(\begin{array}{ccc}
r & 0 & -1 \\
0 & -\beta & 0 \\
-\frac{(1-\alpha) r^{2}}{\alpha \sigma} & -\frac{(1-\sigma) \beta C_{*}}{\sigma E_{*}} & 0
\end{array}\right),
$$

and

$$
B=\left(\begin{array}{ccc}
0 & 0 & 0 \\
-\gamma r & 0 & 0 \\
-\frac{(1-\sigma) \gamma r C_{*}}{\sigma E_{*}} & 0 & 0
\end{array}\right) .
$$

A direct calculation leads to

$$
\Delta(\xi)=\xi^{3}+a_{2} \xi^{2}+a_{1} \xi+a_{0}+e^{-\xi \tau}\left(b_{1} \xi\right)=0,
$$

where

$$
\begin{aligned}
& a_{2}=\beta-r \gtreqless 0, \quad a_{1}=-\left[\frac{(1-\alpha) r^{2}}{\alpha \sigma}+r \beta\right]<0, \\
& a_{0}=-\frac{(1-\alpha) \beta r^{2}}{\alpha \sigma}<0, \quad b_{1}=-\frac{(1-\sigma) \gamma r C_{*}}{\sigma E_{*}} \lessgtr 0 .
\end{aligned}
$$

In order to study the local stability of the equilibrium of system (8) we need to investigate the distribution of the roots of (12). As a control variable enters the problem, it is important to clarify the concept of stability. We say that the equilibrium is completely stable (that is, indeterminate) if all roots have negative real parts; it is a saddle if there exist at least two roots whose real parts have different sign; it is saddlepath stable if there exists a unique root with positive real part; it is unstable if all the roots have positive real part. These forms of stability markedly differ with respect to the ones introduced by Bambi (2008). This is because in his specification of the problem there were both retarded and advanced variables (lags and leads) in the optimality conditions (see Tsuzuki, 2014, for a modelling approach similar to the one used in the present article).

When $\tau=0$, Eq. (12) reduces to

$$
P(\xi)=\xi^{3}+a_{2} \xi^{2}+\left(a_{1}+b_{1}\right) \xi+a_{0}=0 .
$$

Since $P(\xi)=+\infty$ as $\xi \rightarrow+\infty$ and $a_{0}<0$, we conclude that Eq. (13) has at least one positive root. Therefore, the system cannot be completely stable. In particular, we have the following result: 
Proposition 1 Let $\left(C_{*}, K_{*}, E_{*}\right)$ be defined in (9). If

$$
a_{1}+b_{1}=\left[\alpha^{\frac{1}{1-\alpha}} \gamma(\sigma-1) r^{\frac{1-2 \alpha}{1-\alpha}}+E^{*} r((r-\beta \sigma) \alpha-r)\right] / \sigma E^{*} \alpha<0
$$

or $\sigma \in(0,1)$ or $r<\beta$ then the system is saddlepath stable, i.e. there exists a unique twodimensional manifold which determines the set of trajectories converging to the steady state.

We note that if the hypotheses of Proposition 1 do not hold, the system can fall into any of the above cases just described. Therefore, the dynamics of the model can be characterised by persistent fluctuations. These fluctuations are avoided if the environmental good and the private good are complementary between each other.

Consider now the dynamic properties of the model for a positive value of the time delays. Let $\tau>0$. If $\xi=i \omega(\omega>0)$ is a root of Eq. (12), then $\omega$ satisfies

$$
-\omega^{3} i-a_{2} \omega^{2}+a_{1} \omega i+a_{0}+b_{1} \omega i(\cos \omega \tau-i \sin \omega \tau)=0 .
$$

Separating the real and imaginary parts, we have

$$
a_{2} \omega^{2}-a_{0}=b_{1} \omega \sin \omega \tau, \quad-\omega^{3}+a_{1} \omega=-b_{1} \omega \cos \omega \tau .
$$

By taking square on both sides of Eq. (14) and summing them up, it follows that

$$
\omega^{6}+p \omega^{4}+q \omega^{2}+s=0,
$$

where

$$
\begin{aligned}
& p=a_{2}^{2}-2 a_{1}=(\beta-r)^{2}+2\left[\frac{(1-\alpha) r^{2}}{\alpha \sigma}+r \beta\right]>0, \\
& q=a_{1}^{2}-2 a_{0} a_{2}-b_{1}^{2}=\left[\frac{(1-\alpha) r^{2}}{\alpha \sigma}+r \beta\right]^{2}+2 \frac{(1-\alpha) \beta r^{2}}{\alpha \sigma}(\beta-r)-\left[\frac{(1-\sigma) \gamma r C_{*}}{\sigma E_{*}}\right]^{2}, \\
& s=a_{0}^{2}=\left[\frac{(1-\alpha) \beta r^{2}}{\alpha \sigma}\right]^{2}>0 .
\end{aligned}
$$

We note that $q<0$ can hold when the dynamic system without delays is saddlepath stable (this can be verified by using the parameter set: $\bar{E}=10, \gamma=0.2, r=0.003, \alpha=0.13, \beta=0.05$ and $\sigma=0.17)$ as well as when the fixed point is unstable, i.e., all the eigenvalues have positive real part (this can be verified by using the parameter set: $\bar{E}=13, \gamma=0.042, r=0.04, \alpha=0.3$, $\beta=0.01$ and $\sigma=20.7)$.

Now, let $z=\omega^{2}$. then Eq. (15) can be rewritten as

$$
Q(z)=z^{3}+p z^{2}+q z+s=0 .
$$

\section{Lemma 2}

1) If $q \geq 0$, Eq. (17) has no positive solutions.

2) If $q<0$, Eq. (17) has one positive solution $z_{0}=z_{*}=\left(-p+\sqrt{p^{2}-3 q}\right) / 3$ if $Q\left(z_{*}\right)=0$, where $z_{*}=\left(-p+\sqrt{p^{2}-3 q}\right) / 3$, and two positive solutions, say $z_{1}$ and $z_{2}$, with $z_{1}<z_{2}$, if $Q\left(z_{*}\right)<0$. In addition, notice that $Q^{\prime}\left(z_{1}\right)<0$ and $Q^{\prime}\left(z_{2}\right)>0$. 
Proof. If $q \geq 0$, it is immediate that Eq. (17) has no positive roots. If $q<0$, an application of Descartes' rule of signs implies that Eq. (17) has two positive roots, say $z_{1}$ and $z_{2}$, or no positive roots. The last part of the statement follows by noting that $Q(0)=s>0, Q(+\infty)=+\infty$, $Q^{\prime}(z)=3 z^{2}+2 p z+q, Q^{\prime \prime}(z)=6 z+2 p z>0 ; Q(z)$ is a convex function with a minimum at $z_{*}=\left(-p+\sqrt{p^{2}-3 q}\right) / 3$.

Let $q<0$. From the previous Lemma, we know that the characteristic equation (12) has a pair of purely imaginary roots of the form

$$
\xi= \pm i \omega_{k}, k=0,1,2, \text { with } \omega_{k}=\sqrt{z_{k}} .
$$

From (14), we have

$$
\tan \omega \tau=\frac{a_{2} \omega^{2}-a_{0}}{\omega^{3}-a_{1} \omega}
$$

Thus, by solving for $\tau$ we get the corresponding $\tau_{k}^{(j)}(j=0,1,2, \ldots)$

$$
\tau_{k}^{(j)}= \begin{cases}\frac{1}{\omega_{k}}\left\{\tan ^{-1}\left[\frac{a_{2} \omega_{k}^{2}-a_{0}}{\omega_{k}^{3}-a_{1} \omega_{k}}\right]+2 j \pi\right\}, & \text { if } a_{2} \omega_{k}^{2}-a_{0}>0, \\ \frac{1}{\omega_{k}}\left\{\tan ^{-1}\left[\frac{a_{2} \omega_{k}^{2}-a_{0}}{\omega_{k}^{3}-a_{1} \omega_{k}}\right]+(2 j+1) \pi\right\}, & \text { if } a_{2} \omega_{k}^{2}-a_{0}<0 .\end{cases}
$$

Let $\xi(\tau)=\nu(\tau)+i \omega(\tau)$ denote the roots of (12) near $\tau=\tau_{k}^{(j)}$ satisfying $\nu\left(\tau_{k}^{(j)}\right)=0$ and $\omega\left(\tau_{k}^{(j)}\right)=\omega_{k}$.

Proposition $3 \xi= \pm i \omega_{k}, k=1,2$, are simple roots of (12) when $\tau=\tau_{k}^{(j)}$ and one has the following transversality conditions

$$
\left[\frac{d \operatorname{Re}(\xi)}{d \tau}\right]_{\tau=\tau_{1}^{(j)}, \omega=\omega_{1}}<0 \quad \text { and } \quad\left[\frac{d \operatorname{Re}(\xi)}{d \tau}\right]_{\tau=\tau_{2}^{(j)}, \omega=\omega_{2}}>0
$$

Proof. Differentiating Eq. (12) with respect to $\tau$, we obtain

$$
\left[3 \xi^{2}+2 a_{2} \xi+a_{1}+b_{1}(1-\tau \xi) e^{-\xi \tau}\right] \frac{d \xi}{d \tau}=b_{1} \xi^{2} e^{-\xi \tau}
$$

Therefore

$$
\left(\frac{d \xi}{d \tau}\right)^{-1}=\frac{\left(3 \xi^{2}+2 a_{2} \xi+a_{1}\right) e^{\xi \tau}}{b_{1} \xi^{2}}+\frac{1}{\xi^{2}}-\frac{\tau}{\xi}
$$

By using (12), we get

$$
\begin{aligned}
\operatorname{sign}\left\{\left.\frac{d(\operatorname{Re} \xi)}{d \tau}\right|_{\tau=\tau_{k}^{(j)}, \omega=\omega_{k}}\right\} & =\operatorname{sign}\left\{\operatorname{Re}\left(\frac{d \xi}{d \tau}\right)_{\tau=\tau_{k}^{(j)}, \omega=\omega_{k}}^{-1}\right\}=\operatorname{sign}\left\{\frac{3 \omega_{k}^{4}+2 p \omega_{k}^{2}+q}{b_{1}^{2} \omega_{k}^{2}}\right\} \\
& =\operatorname{sign}\left\{3 z_{k}^{2}+2 p z_{k}+q\right\}=\operatorname{sign}\left\{Q^{\prime}\left(z_{k}\right)\right\} .
\end{aligned}
$$

Next, we prove that $\pm i \omega_{k}$ are simple roots. By contradiction, if, for example, $\xi=i \omega_{k}$ is a repeated root of $(12)$, then the derivative of (12) with respect to $\xi$ evaluated at $\xi=i \omega_{k}$ should be zero, i.e. $\left[3 \xi^{2}+2 a_{2} \xi+a_{1}+b_{1}(1-\tau \xi) e^{-\xi \tau}\right]_{\xi=i \omega_{k}}=0$. Thus, (19) would lead to $\left[b_{1} \xi^{2} e^{-\xi \tau}\right]_{\xi=i \omega_{k}}=0$, i.e. $\cos \omega_{k}=0$ and $\sin \omega_{k}=0$., which is a contradiction. Similar argument for $\xi=-i \omega_{k}$. This completes the proof. 
Remark 4 If $\xi= \pm i \omega_{0}$, i.e. $Q(z)$ has only one positive solution, then the transversality condition does not hold being $Q^{\prime}\left(z_{0}\right)=0$.

According to the previous analysis we have the following results on the stability of the equilibrium of system (8).

Theorem 5 Let $\left(C_{*}, K_{*}, E_{*}\right), q, \tau_{k}^{(j)}(k=1,2)$ be defined as in $(9),(16),(18)$, respectively, and assume (10) holds.

1) If $q \geq 0$ then the equilibrium $\left(C_{*}, K_{*}, E_{*}\right)$ is saddlepath stable for all $\tau \geq 0$.

2) If $q<0$ and the equilibrium $\left(C_{*}, K_{*}, E_{*}\right)$ is saddlepath stable for $\tau=0$ then there exist stability switches for $\tau>0$ if $Q\left(z_{*}\right)<0$. Furthermore, system (8) undergoes a Hopf bifurcation at $\left(C_{*}, K_{*}, E_{*}\right)$ when $\tau=\tau_{k}^{(j)}(j=0,1,2, \ldots)$.

By comparing the dynamic properties classified in Proposition 1 (related to the model without time delays) with those classified in Theorem 5 (related to the model with time delays), it is possible to note that the existence of a time delay within the evolutionary dynamics of environmental quality can cause cyclical dynamics also in the case of complementarity between consumption of the private good and the environmental good (see Figure 1). This result brakes with respect to modelling approaches on environmental issues developed in continuous time without delays (Antoci et al., 2007) as well as in an OLG setting (Antoci et al., 2016), where the possibility of non-monotonic dynamics occurs only when the private good and the environmental good are substitutes.

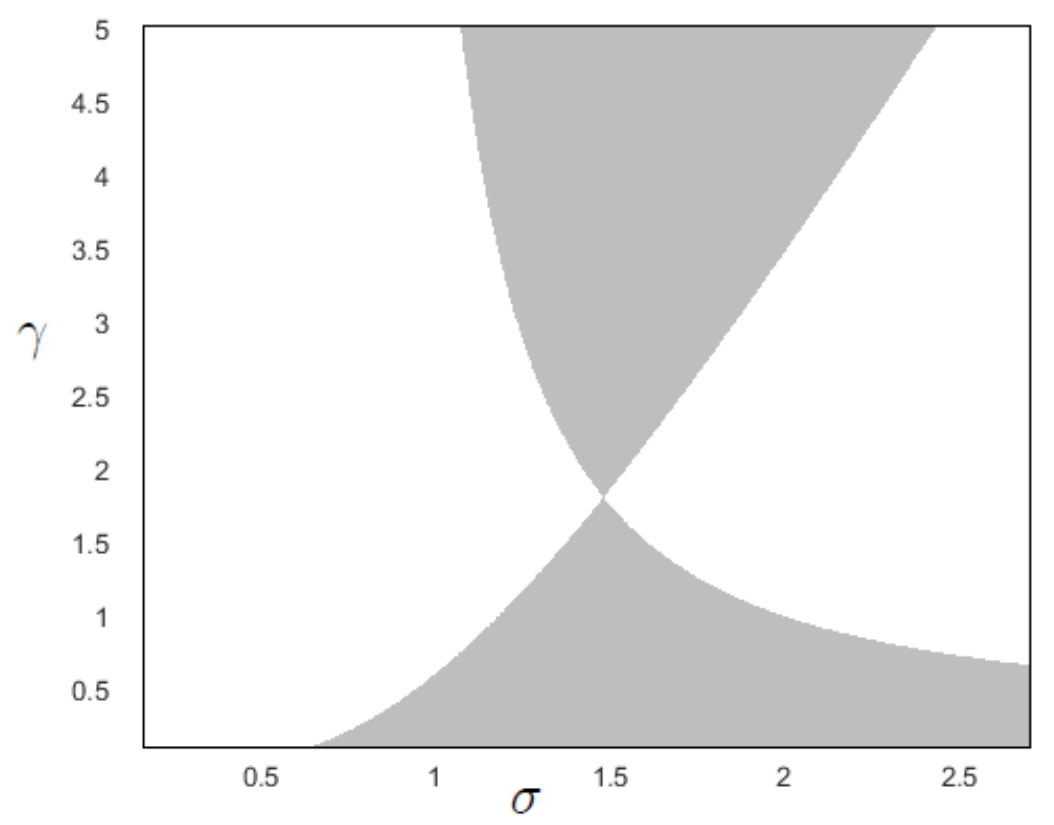

Figure 1. Parameter set: $r=0.03, \alpha=0.33, \beta=0.9$ and $\bar{E}=10$. The grey region shows the set of couples $(\sigma, \gamma)$ such that the system (by starting from a context of saddle path stability) undergoes a Hopf bifurcation as long as the time delay $\tau$ increases. 


\section{Conclusions}

The importance of the environment has been increasingly acknowledged in academic circles. This is particularly the case for the relationship between environmental quality and economic growth. Economists are thus faced with the great challenging of thinking and building on models able to capture the essence of the sustainable development. Within this line of research, this article has studied a model in which the environment enters the utility function of economic agents as a public good. The environment, in turn, is affected by the production activity driven by the allocating choices of the agents in a decentralised economy. The work has characterised the dynamics of a continuous time model with and without time lags in the effects of the economic activity on environmental resources. In the absence of time delays, the model is saddlepath stable if private consumption and the environmental good are complements. In this case, cyclical behaviours can occur only in the case of substitutability between consumption of the private good and the environmental good. Instead, with a positive time delay it is possible to have cyclical behaviours also when private consumption and the environmental good are substitutes. It is important to note that the way in which time delays are introduced in the present work (acting on the evolution of the environmental externality) generate dynamic phenomena similar to those obtained in a context where a technology with time to build or vintage capital is employed.

Acknowledgements The authors gratefully acknowledge three anonymous reviewers for comments and suggestions allowing an improvement in the quality of the article. The usual disclaimer applies.

Conflict of Interest The authors declare that they have no conflict of interest.

\section{References}

[1] Antoci, A., 2009. Environmental degradation as engine of undesirable economic growth via self-protection consumption choices. Ecological Economics 68, 1385-1397.

[2] Antoci, A., Galeotti, M., Russu, P., 2007. Undesirable economic growth via agents' selfprotection against environmental degradation. Journal of the Franklin Institute 344, 377390.

[3] Antoci, A., Galeotti, M., Russu, P., 2011. Poverty trap and global indeterminacy in a growth model with open-access natural resources. Journal of Economic Theory 146, 569-591.

[4] Antoci, A., Gori, L., Sodini, M., 2016. Nonlinear dynamics and global indeterminacy in an overlapping generations model with environmental resources. Communications in Nonlinear Science and Numerical Simulation 38, 59-71.

[5] Ayong Le Kama, A.D., 2001. Sustainable growth, renewable resources and pollution. Journal of Economic Dynamics \& Control 25, 1911-1918.

[6] Bambi, M., 2008. Endogenous growth and time-to-build: the AK case. Journal of Economic Dynamics \& Control 32, 1015-1040.

[7] Bambi, M., Gori, F., 2014. Unifying time-to-build theory. Macroeconomic Dynamics 18, $1713-1725$. 
[8] Benhabib, J., Rustichini, A., 1991. Vintage capital, investment, and growth. Journal of Economic Theory 55, 323-339.

[9] Boucekkine, R., Germain, M., Licandro, O., 1997. Replacement echoes in the vintage capital growth model. Journal of Economic Theory 74, 333-348.

[10] Boucekkine, R., de la Croix, D., Licandro, O. 2011. Vintage capital growth theory: three breakthroughs. In: de La Grandville, O. (ed.) Economic Growth and Development (Frontiers of Economics and Globalization, Volume 11) Emerald Group Publishing Limited, Chapter $5,87-116$.

[11] Boucekkine, R., Germain, M., Licandro, O., Magnus, A., 1998. Creative destruction, investment volatility and the average age of capital. Journal of Economic Growth 3, 361-384.

[12] Boucekkine, R., Licandro, O., Puch, L., del Rio, F., 2005. Vintage capital and the dynamics of the AK model. Journal of Economic Theory 120, 39-72.

[13] Bovenberg, A.L., Smulders, S., 1995. Environmental quality and pollution-augmenting technological change in a two-sector endogenous growth model. Journal of Public Economics 57, $369-391$.

[14] Brock, W.A., Scott Taylor, M., 2010. The Green Solow model. Journal of Economic Growth $15,127-153$.

[15] d'Albis, H., Augeraud-Véron, E., Hupkes, H.J., 2014. Multiple solutions in systems of functional differential equations. Journal of Mathematical Economics 52, 50-56.

[16] Caulkins, J.P., Hartl, R.F., Kort, P.M., 2010. Delay equivalence in capital accumulation models. Journal of Mathematical Economics 46, 1243-1246.

[17] D'Alessandro, S., 2007. Non-linear dynamics of population and natural resources: the emergence of different patterns of development. Ecological Economics 62, 473-481.

[18] Day, R.H., 1982. Irregular growth cycles. American Economic Review 72, 406-414.

[19] Gori, L., Guerrini, L., Sodini, M., 2015. Hopf bifurcation and stability crossing curves in a cobweb model with heterogeneous producers and time delays. Nonlinear Analysis: Hybrid Systems 18, 117-133.

[20] Hartl, R.F., Kort, P.M., 2010. Delay in finite time capital accumulation. Central European Journal of Operations Research 18, 465-475.

[21] Kalecki, M., 1935. A macroeconomic theory of business cycles. Econometrica 3, 327-344.

[22] Itaya, J.I., 2008. Can environmental taxation stimulate growth? The role of indeterminacy in endogenous growth models with environmental externalities. Journal of Economic Dynamics \& Control 32, 1156-1180.

[23] John, A., Pecchenino, R., 1994. An overlapping generations model of growth and the environment. Economic Journal 104, 1393-1410.

[24] Mapanda, F., Mangwayana, E.N., Nyamangara,J., Giller, K.E., 2005. The effect of longterm irrigation using wastewater on heavy metal contents of soils under vegetables in Harare, Zimbabwe. Agriculture, Ecosystems and Environment 107, 151-165. 
[25] Matsumoto, A., Szidarovszky, F., 2012. Nonlinear delay monopoly with bounded rationality, Chaos Solitons Fractals 45, 507-519.

[26] Nadadur, S.S. Hollingsworth, J.W., 2015. Air Pollution and Health Effects. Springer-Verlag, London.

[27] Solow, R.M., 1956. A contribution to the theory of economic growth. Quarterly Journal of Economics 70, 65-94.

[28] Tsuzuki, E., 2014. A new Keynesian model with delay: Monetary policy lag and determinacy of equilibrium. Economic Analysis and Policy 44, 279-291.

[29] Wirl, F., 1997. Stability and limit cycles in one-dimensional dynamic optimisations of competitive agents with a market externality. Journal of Evolutionary Economics 7, 73-89.

[30] Wirl, F., 2004. Sustainable growth, renewable resources and pollution: thresholds and cycles. Journal of Economic Dynamics \& Control 28, 1149-1157.

[31] Xepapadeas, A., 2005. Economic growth and the environment. In Mäler, K.G., Vincent, J.R., Eds., Handbook of Environmental Economics, Vol. 3, Ch. 23, 1219-1271.

[32] Zhang, J., 1999. Environmental sustainability, nonlinear dynamics and chaos. Economic Theory 14, 489-500. 